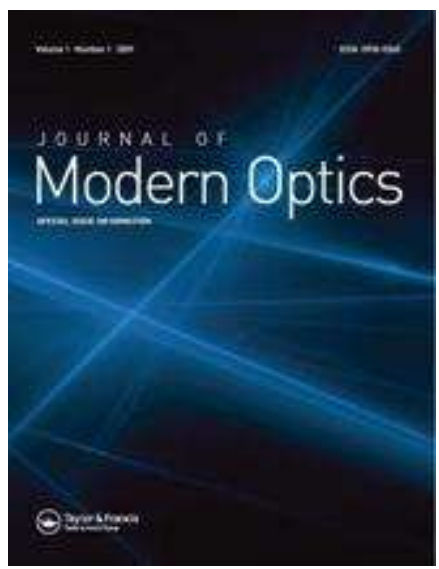

\title{
Blinking kinematics description through non invasive measurement
}

\begin{tabular}{|c|c|}
\hline Journal: & Journal of Modern Optics \\
\hline Manuscript ID: & TMOP-2010-0589.R1 \\
\hline Manuscript Type: & Special Issue Paper - Vision Science and Ophthalmic Optics \\
\hline $\begin{array}{l}\text { Date Submitted by the } \\
\text { Author: }\end{array}$ & 23-Dec-2010 \\
\hline Complete List of Authors: & $\begin{array}{l}\text { Perez, Jorge; Universidad de Alicante; Universidad de Alicante, } \\
\text { Optica } \\
\text { Espinosa, Julian; Universidad de Alicante, Optica } \\
\text { Domenech, Begoña; Universidad de Alicante, Optica } \\
\text { Mas, David; Universidad de Alicante, Optica } \\
\text { Illueca, Carlos; Universidad de Alicante, Optica }\end{array}$ \\
\hline Keywords: & eye blinking, image processing, mathematical models \\
\hline \multicolumn{2}{|c|}{$\begin{array}{l}\text { Note: The following files were submitted by the author for peer review, but cannot be converted } \\
\text { to PDF. You must view these files (e.g. movies) online. }\end{array}$} \\
\hline Figure2.flv & \\
\hline
\end{tabular}

\section{SCHOLARONE \\ Manuscripts}


Blinking kinematics description through non invasive measurement

Jorge Perez, Julian Espinosa, Begoña Domenech, David Mas*, Carlos

Illueca

Department of Optics, University of Alicante, Alicante, Spain

P.O. Box, 99, 03080, Alicante.

*corresponding author: david.mas@ua.es 


\title{
Blinking kinematics description through non invasive measurement
}

\begin{abstract}
Spontaneous eye blinking is a complex process which implies the joint actions of the eyelid muscles and the eye globe. These actions provoke the eyelid closure and, simultaneous eye retraction into the orbit. Recently, the authors presented a non-invasive technique for measuring both movements that permits calculating several parameters. Following the work from Malbouisson et al., we have fitted the displacement curves of the eyelid to an analytical function and, additionally, we have extended the kinematic model to the eye retraction movements. As a result of the fitting, some dynamic parameters have been presented for the first time in the literature. This study can be easily extended to obtain relevant information about ocular biomechanical properties in physiological optics and provides results that may be of interest in neurophysiology, in order to understand the blinking mechanism
\end{abstract}

Keywords: eye blinking, image processing, mathematical modelling

\section{Introduction and purpose}

Blinking is the fast movement of closing and opening of the eyelid. It is a complex biomechanical process essential in the tear film distribution over the ocular surface [12]. It is accepted that the blink is not a simple process of eyelid movement but a joint action of the palpebral muscles and the eye globe [3]. Aside of the lids closure, when the blinking process starts, the eye moves in the inferior nasal-ward direction while it performs a cyclotorsional rotation [4-7]. Simultaneously with these movements, the eye performs a retraction inside the orbit of $0.5-1.5 \mathrm{~mm}$ [4,6-8]. All these eye movements are in principle associated to a co-contraction of majority of extra-ocular muscles although the mechanism is still not fully understood [3,7]. As a consequence of that, every blink produces a momentary suppression of vision [9].

Blinking dynamics and its relation with eye movements have been traditionally addressed from the field of neurophysiology [10]. Registration of the eye activity is usually done through a magnetic search coil technique. Although this method is considered as the gold standard in eye movements recordings [11], the recording time is limited since it is invasive and provokes great discomfort in the eye. 
Eyelid position is determined through an additional coil on the upper lid $[3,8,12]$. In spite of this method is less invasive, the presence of a strange body attached to the lid may change the blinking pattern.

Recent technological advances have produced non-invasive image based techniques for detecting eye movement during blinking, like those based in high speed video capture [13-16]. In [6], the authors measure the lid position and the eye globe retraction with a photographic sequence at 48 to 74 frames per second (fps). Choi et al. [15] also used a high speed camera at $60 \mathrm{fps}$ to dynamically measure the eyelid motion. Sampling rate limitation and the difficulty to obtain quantitative data are serious drawbacks to the use of image based techniques. Fortunately, new devices and algorithms have led to systems with accuracy comparable to the search coil methods, and thus, it is expected that in near future these methods will not be restricted to clinical labs [17].

In a previous work, our group has presented a non invasive technique for high speed measuring some of the dynamic processes that occur during blinking [18]. In particular, the technique allows simultaneously measuring the corneal retraction and the eyelid motion during a spontaneous blink. Preliminar results and some dynamic parameters linked to the eye blinking were published there, although some additional data are given here.

Mathematical modelling of eyelid trajectory has been recently introduced [19] but few quantitative results were given by the authors. Based on that work, we propose here an analytical model of the eye blinking including lid movement and ocular retraction. We provide new numerical results and pose some new hypothesis. These results may be of interest in neurophysiology, in order to understand the 
blinking mechanism, and in physiological optics, in order to obtain relevant information about ocular biomechanical properties.

\section{Corneal retraction and eyelid motion measurements}

The setup for illuminating, capturing and processing the image of the anterior corneal segment in our experiment was widely described in a previous work [18]. Briefly, the system consists on a slit lamp and an external fast camera working at 500 frames per second. In figure 1, we show a picture of a subject being explored. The reader can see the disposal of the different elements, i.e. a slit projection head and videocamera with a zoom lens. A video sequence of one complete blink is shown in figure 2. One can clearly see the simultaneous acquisition of the eye retraction and lid position. The initial corneal position has been marked with a small cross, in order to facilitate the interpretation of the sequence.

Lid displacement was monitored by studying the saturation of the frames in the sequence. The sensitivity of the camera is adjusted to detect the light diffused from the cornea, thus, light diffused by the eyelid saturates the sensor. We assume that the frame with more saturated pixels corresponds to a closed eye state. By counting the number of saturated pixels in each frame, we can reconstruct the position of the eyelid.

Eye retraction is evaluated by analyzing a scanning line perpendicular to the inferior part of the cornea, at the cross position in figure 2 . The presence of the cornea is detected by binarizing the image and detecting the presence of a white to black border. Proper pixel to millimetre calibration of the image permits the measurement of the eye retraction.

\section{Mathematical models}


The physiological mechanisms controlling the blink dynamics are known. Upper eyelid motion starts when the Levator Palpebral Superioris muscle (LPSM) is abruptly inhibited and the eyelid performs a passive movement downwards. Simultaneous to the LPSM inhibition, the Orbicularis Oculi muscle (OOM) starts a fast contraction thus producing a strong active force. This combination of inhibition and activation of muscular action produces the closure phase of the eyelid. After the action of the OOM, the LPSM starts a restoration force which pulls up the lid to its initial position. Nevertheless, when the OOM action ceases, the upper lid keeps its movement down due to its inertia. The acceleration of the movement in the opposite direction makes that the absolute value of the movement velocity is slowly decreasing until the movement is reversed (see Figure 3).

According to the forces acting on the eyelid, we divide the blink process in two parts, these being different from the typical closing and opening phases. The first one, which is called here as active part, goes from the start to the point of maximum absolute velocity and coincides with the active muscular action. The second part can be defined as the recovery part and covers the time from the peak velocity to the end of the blinking, being in this part when the forces pull back the lid to its original position. In other words, both parts are defined by the sign of the eyelid acceleration.

In [19], the authors assume that the OOM contraction is similar to a muscle jerk [20] and propose an analytical expression for the acceleration (or equivalently impulsive force per unit mass) in the form:

$$
a(t)=b t(t-\tau)
$$

The OOM action starts at $t=0$ and finishes at $t=\tau$, being this last moment when the absolute eyelid velocity reaches its highest value (Figure 3). The position of the eyelid during this period can be written as: 


$$
X_{1}(t)=\frac{1}{12} b t^{3}(t-2 \tau)
$$

After ceasing OOM, there is a short time when the lid moves down under the action of the LPSM and then, it goes back to its initial position. This last phase starts at the point with maximum velocity and can be described as a damped oscillation:

$$
X_{2}(t)=A \exp (-\mu t) \cos (\omega t+\phi)
$$

The parameters $b, \tau, \omega$ and $\mu$, respectively represent the strength of the muscle force, its actuation time, frequency of the oscillation and the attenuation constant of the system and will be experimentally adjusted. Although the rest of parameters also represent physical magnitudes of the system, we do not consider them relevant for the movement description.

Data from eyelid movement of 6 subjects have been fitted to the above mathematical model. The procedure has been the following: data from each blinking has been fitted to the analytical function which provides the smallest fitting error. This function had to be continuous and derivable during one blinking period. The fitting function has not any particular physical meaning; however it is used here just for determining the instant of maximum absolute velocity, which happens at $t=\tau$. (see [19] for more details). This time match the connection point between equations (2) and (3). Once this point is determined, we perform the fitting obtaining the following results (mean \pm standard deviation):

$$
\begin{aligned}
& b_{l}=(11.7 \pm 15.4) \times 10^{3} \mathrm{~mm} / \mathrm{s}^{4} \\
& \tau_{l}=(51.1 \pm 16.5) \mathrm{ms} \\
& \mu_{l}=10.8 \pm 2.4 \mathrm{~s}^{-1} \\
& \omega_{l}=12.5 \pm 2.6 \mathrm{~s}^{-1}
\end{aligned}
$$

being the average correlation coefficient of the first and second parts (active and recovery phases) respectively $r_{a}^{2}=0.96 \pm 0.04$ and $r_{r}^{2}=0.98 \pm 0.04$. Subscript $l$ 
stands for lid. In figure 3, a typical case of the fitting functions for one observer is depicted

We would like to focus the reader's attention on the plateau observed in the eyelid graph. This plateau does not seem to appear in results from other authors when coils are used [19], although other authors using imaging technique observe such phenomena [13]. Our hypothesis is that when the eyelid closes the eye, the skin is still moving and this is detected by the search coil. In our case, since there is not displacement of the lid, the saturation does not change and thus, a plateau is registered. The duration of this planar region informs us about the time that the eye is completely closed and even allows detecting whether the blink has been complete or not. As can be appreciated, the data in that region do not fit well to the proposed curves. The presence of this region diminishes the accuracy of the fitting, but does not affect to the obtained parameters.

The mechanism for eye retraction inside the orbit is not so well known. The most accepted hypothesis is that simultaneous contraction of extraocular muscles is responsible of ocular retraction and ciclotorsion [4]. Restitution of the eye to its original position is due to muscular relaxation and no additional pulling force is required to explain such movement. Up to our knowledge, no analytical function describing eye retraction has been proposed. Although our method does not permit visualization of the whole eye trajectory, data describing the position of the anterior cornea with time suggest a mathematical description similar to the one acting in the lid, with a pulling force and then a restitution force. We assume the presence of an active and a recovery phases. Thus, the process is divided in two parts according to the sign of the acceleration. We adjust data to the equations (2) and (3) and expect to obtain physical parameters describing the eye retraction. 
The performance of the fitting of the eye retraction data presents an important drawback since part of the data is lost due to eyelid covering (see Figure 2 and 4). This fact impedes the determination of the point with maximum velocity and thus, the matching point between the two. In order to fit experimental values, we approximated this point to the last visible point. According to figures 3 and 4, matching between active and recovery phases resulted to be at the same time, but, while at that moment the eyelid has started the recovery phase, the eye is still under the effect of a pulling force. This indeed introduces an error. Nevertheless, since majority of data points are well determined, we assume that the obtained results are correct.

After fitting of all data, we noticed that assuming acceleration in the form of (1) provided contradictory results. Although we obtained good correlation coefficients, many of the results had no physical sense $(\tau<0)$. As we stated above, the premature eye occlusion by the eyelid only allowed capturing part of the active phase, being lost the connection with the recovery one. During the observed active phase any of the observed eyes reached the maximum value of the absolute velocity or even changes its tendency. Therefore, when the eye is occluded the acceleration may be still increasing so it can be described as:

$$
a(t)=b t^{2} \quad \rightarrow \quad X_{1}(t)=\frac{b}{12} t^{4}
$$

This assumption means that the active phase may be significant longer for the eye that for the lid. Taking into account all these considerations, the obtained parameters have been:

$$
\begin{aligned}
& b_{e}=(-0.488 \pm 0.471) \times 10^{3} \mathrm{~mm} / \mathrm{s}^{4} \\
& \mu_{e}=17.0 \pm 2.7 \mathrm{~s}^{-1} \\
& \omega_{e}=2.2 \pm 0.9 \mathrm{~s}^{-1}
\end{aligned}
$$


with correlation coefficients $r_{a}^{2}=0.96 \pm 0.03$ and $r_{r}^{2}=0.97 \pm 0.02$ for the active and recovery phases, respectively.

\section{Discussion}

Eye lid motion is usually analyzed through parameters such as amplitude, average and maximum velocity of both up and down phases. In Table 1, we present our results directly measured from the experimental data together with those in the bibliography $[6,8,12,14,16,19-23]$. Data should be interpreted as mean values \pm standard deviation. One can see there that our results are in reasonable agreement with results from the bibliography, being the discrepancy inside the statistic error. It may be surprising the high standard deviation obtained by us and the majority of authors. This fact is due to averaging between different subjects. When blinks from only one single observer are considered, standard deviation in majority of results is reduced to $10 \%$.

Many parameters obtained in (4) and (6) are also new in the literature. Malbouisson et al. [19] give values for the eyelid active phase duration, $\tau_{l}$, giving values between 70 and $80 \mathrm{~ms}$. These values are slightly higher than those here obtained. Differences may come from setting the starting point of the blink, but according to the high inter-subject dispersion obtained in this kind of measurements, we find that differences are not significant.

Value obtained for $b_{l}$ may seem really dispersive; however, a careful look of the data shows that the highest values obtained for $b_{l}$ correspond to the shortest values obtained for $\tau_{l}$. If we consider the absolute value of the mechanical impulse per unit mass instead of the force exerted on the eyelid, we obtain:

$$
|I|=\left|\int_{0}^{\tau} F(t) d t\right|=\frac{b_{l} \tau^{3}}{6} \quad \rightarrow \quad I=(118 \pm 53) \times 10^{-3} \mathrm{~N} \cdot \mathrm{s}
$$


what means that the intensity of the muscular forces acting on the eyelid are closely correlated with the duration time of this action. Therefore, the increment of the mechanical momentum of the eyelid is approximately constant during the active phase.

Although eye retraction is a known phenomenon, there are few works that quantify it during blinking. The related bibliography [1,4,6,23] establishes this retraction in 1-1.5 mm. Collins et al. distinguished between four different eye lid fissure conditions (widening, blink, gentle close and hard close) with different associated eye retraction. In our case, we only selected gentle (spontaneous) blinks.

Our method [18] does not allow complete visualization of the eye retraction phenomenon. However, the fitting function (see Figure 4) permits completing the trajectory of the eye and calculating the maximum eye retraction. From all fittings, we obtained an average retraction of $1.2 \pm 0.4 \mathrm{~mm}$ which is in agreement with [1]. Analysis of fitting function also permits estimating the maximum eye velocity of eye retraction giving $37.4 \pm 16.8 \mathrm{~mm} / \mathrm{s}$. This result is new in the literature and should be confirmed by further analysis.

The reader should notice that the damping constant obtained for the cornea is higher than that for the eyelid, so recovery time should be shorter. It should be also noticed that the whole blinking process takes more time in the eye that in the eyelid, as can be deduced from direct measurements in Table 1. This fact confirms our hypothesis that the active phase is larger than the recovery phase, so the peak velocity in the eye is reached later than in the eyelid. Detailed analysis of this delay maybe of importance in neurophysiology in order to analyze the synchronization of the neural blinking centre [3]. Note that these parameters have been presented for the first time and additional research is needed to confirm our results. 


\section{Conclusion}

In this paper we have analyzed the kinematics of an eye blinking. Complete set of results for eyelid motion and eye retraction obtained with a non invasive technique have been presented and are comparable to those reported by other authors in both activities of the blink process. The method is non-invasive and provides accurate results. Moreover, its implementation does not require of any specialized component so it can be reproduced in any research lab.

Curves of displacement of both eye and lid have been mathematically modeled and some dynamic parameters have been presented for the first time in the literature. Repetitiveness of some results shows that our hypotheses are reliable, although further research is necessary on this subject in order to confirm the results here exposed.

This study can be easily extended to the analysis of contact lens behavior during blinking, which can be of importance in designing new compensation elements. Also, obtained parameters can be of interest for analysis of biomechanical modeling of the eye or in neurophysiology due to the deep connection between blinking and neural activity. 


\section{Figure captions}

FIGURE 1: Eye blinking was recorded by illuminating the eye with a Haag-Streit slit-lamp (SL-990). Light was projected on the left eye at $45^{\circ}$ to the nasal part. The images were recorded with a high Speed camera (X_PRI AOS technologies AC) working at $500 \mathrm{fps}$ and a spatial resolution of $800 \times 560 \mathrm{px}$ situated outside the lamp at $45^{\circ}$ to the left temporal part.

FIGURE 2 (link to multimedia file). Animation showing the eyelid movement together with the eye retraction. The cross marks the initial position of the cornea. Graphs in the sides show the position (in $\mathrm{mm}$ ) of both elements

FIGURE 3 Eyelid position and fitting to the model for one typical case. Dashed line shows eyelid velocity during blinking. The vertical line shows the instant where the maximum absolute velocity is achieved being this point the transition between the active phase and the recovery phase.

FIGURE 4 Anterior corneal position and fitting to the model for one typical case. The vertical dashed line marks the link between the two fitting curves corresponding to the active and the recovery phases.

\section{Table captions}

TABLE 1 Summarizing table with parametric values of eye retraction and eyelid motion in different studies 


\section{References}

[1] Doane, M.G. Interaction of eyelids and tears in corneal wetting and the dynamics of the normal human eyeblink. Am. J. Ophthalmol. 1980, 89, 507516.

[2] Kirkwood, B.J. Why do humans blink? A short review. Insight 2006, 31, 1517.

[3] Ongerboer de Visser, B.W.; Bour, L.J. Eye and eyelid movements during blinking: an eye blink centre? Suppl. Clin. Neurophysiol. 2006, 58, 16-25.

[4] Evinger, C; Shaw, M.D.; Peck, C.K.; Manning, K.A.; Baker, R. Blinking and Associated Eye-Movements in Humans, Guinea-Pigs, and Rabbits. $J$. Neurophysiol. 1984, 52,323-339.

[5] Collewijn, H.; Vandersteen, J.; Steinman, R.M. Human-Eye Movements Associated with Blinks and Prolonged Eyelid Closure. J. Neurophysiol. 1985, 54, 11-27.

[6] Riggs, L.A.; Kelly, J.P.; Manning, K.A.; Moore, R.K. Blink-Related EyeMovements. Invest. Ophthalmol. Vis. Sci. 1987, 28, 334-342.

[7] Bour, L.J; Aramideh, M.; De Visser, B.W.O. Neurophysiological aspects of eye and eyelid movements during blinking in humans. J. Neurophysiol. 2000, 83, 166-176.

[8] Bergamin, O.; Bizzarri, S.; Straumann, D. Ocular torsion during voluntary blinks in humans. Invest. Ophthalmol. Vis. Sci. 2002, 43, 3438-3443.

[9] Volkmann, F.C.; Riggs, L.A.; Ellicott, A.G; Moore R.K. Measurements of visual suppression during opening, closing and blinking of the eyes. Vis. Res. 1982, 22, 991-996.

[10] Bour, L,.;Ongerboer de Visser, B.W.; Aramideh, M.; Speelman, J. Origin of eye and eyelid movements during blinking. Mov. Disord. 2002, 17, S30-S32.

[11] Collejwin, H. Eye movement recording. In Vision Research: a Practical Guide to Laboratory Methods; Carpenter RHS; Robson JG, Eds.; Oxford University Press; 1998; 245-285.

[12] Evinger, C., Manning, K.A.; Sibony, P.A. Eyelid Movements - Mechanisms and Normal Data. Invest. Ophthalmol. Vis. Sci. 1991, 32, 387-400.

[13] Tsubota, K.; Hata, S.; Okusawa, Y.; Egami, F.; Ohtsuki, T.; Nakamori, K. Quantitative videographic analysis of blinking in normal subjects and patients with dry eye. Arch. Ophthalmol. 1996, 114, 715-720.

[14] Somia, N.N.; Rash, G.S.; Epstein, E.E.; Wachowiak, M.; Sundine, M.J.; Streme,l R.W.; Barker, J.H.; Gossman, D. A computer analysis of reflex eyelid motion in normal subjects and in facial neuropathy. Clin. Biomech. 2000, 15, 766-771.

[15] Choi, S.H.; Park, K.S.; Sung, M.W.; Kim, K.H. Dynamic and quantitative evaluation of eyelid motion using image analysis. Med. Biol. Eng. Comput. 2003, 41,146-150.

[16] Casse, G.; Sauvage, J.P.; Adenis, J.P.; Robert, P.Y. Videonystagmography to assess blinking. Graefes Arch. Clin. Exp. Ophthalmol. 2007, 245, 1789-1796.

[17] Imai, T.; Sekine, K.; Hattori, K.; Takeda N.; Koizuka, I.; Nakamae, K.; Miura, K.; Fujioka, H.; Kubo, T. Comparing the accuracy of videooculography and the scleral search coil system in human eye movement analysis. Auris Nasus Larynx 2005, 32, 3-9. 
[18] Mas, D.; Domenech, B.; Espinosa, J.; Pérez, J.; Hernández, C.; Illueca, C. Noninvasive measurement of eye retraction during blinking. Opt. Lett. 2010, 35, 1884-1886.

[19] Malbouisson, J.M.C.; Messias, A.; García, D.M.; Cechetti, S.P.; Barbosa, J.C.; Cruz, A.A.V. Modeling upper eyelid kinematics during spontaneous and reflex blinks. J. Neurosci. Methods. 2010, 191, 119-125.

[20] Hill, A.; Howard, J. Alternating relaxation heat in muscle twitches. J. Physiol. 1957, 139, 466-473.

[21] Nakamura, Y.; Matsuda, J.; Suzuki, K.; Toyoda, H.; Hakamata, N.; Shimamoto, T.; Kinoshita, S. Measurement of spontaneous blinks a highspeed blink analyzing system. Nippon Ganka Gakkai Zasshi 2008, 112, 10591067.

[22] Sforza, C.; Rango, M.; Galante, D.; Bresolin, N.; Ferrario, V.F. Spontaneous blinking in healthy persons: an optoelectronic study of eyelid motion. Ophthal. Physiol. Opt. 2008, 28, 345-353.

[23] Collins, M.; Smyth, W.; Seawright, J.; Kelly, S. The synkinesis between antero-posterior eye position and lid fissure width. Clin. Exp. Optom. 1992, $75,38-41$. 


\section{Aknowledgements}

This work has been supported by the Generalitat Valenciana project nr. GV/2009/002 and by the Ministerio de Ciencia e Innovación through the project nr. FIS2009-05639-E/FIS. J. Espinosa acknowledges the support of the Generalitat Valenciana through the project BEST/2010/209. D. Mas acknowledges the support of the Ministerio de Educacion through the project PR2009-0377 


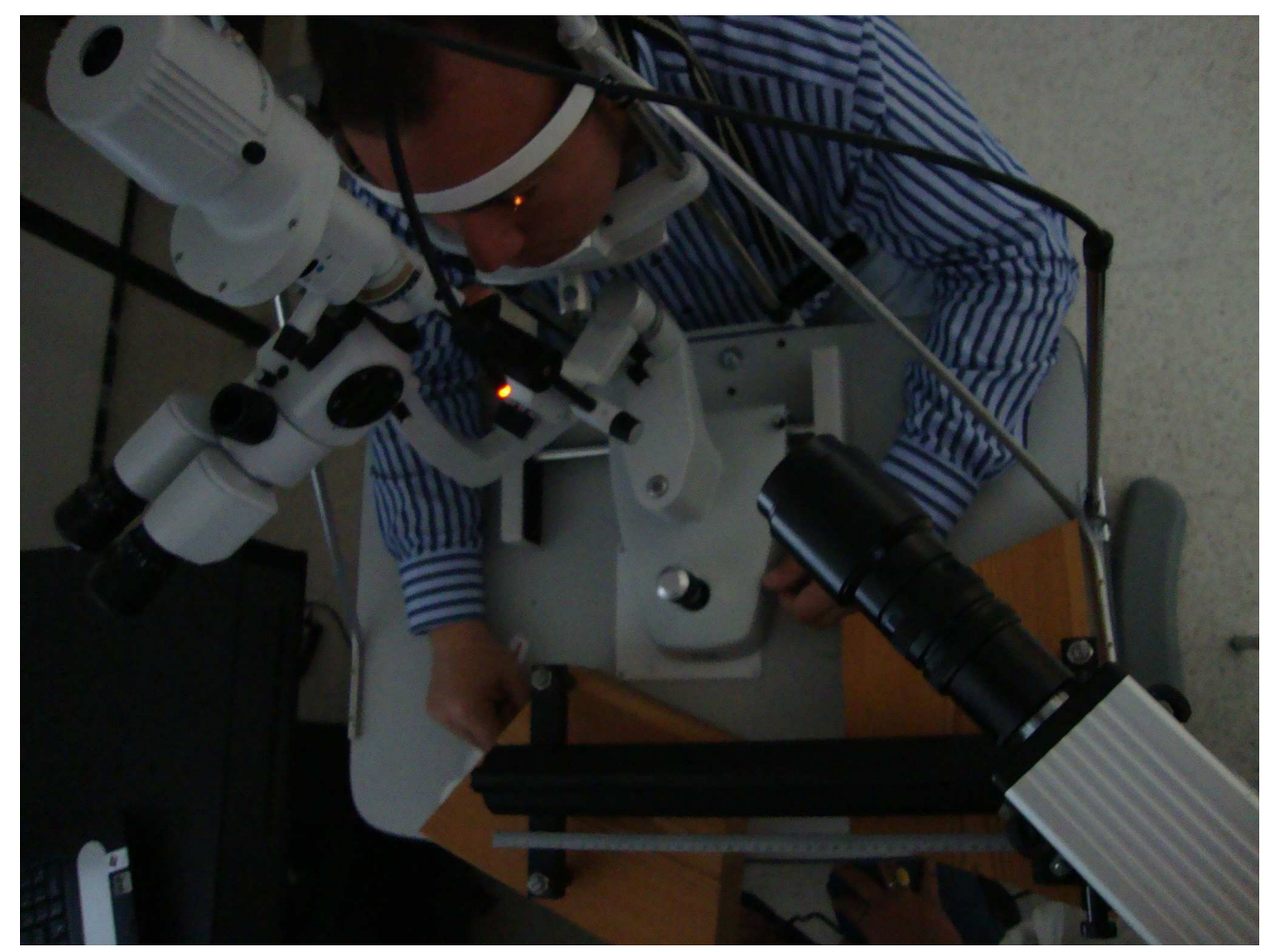

Figure 1. Eye blinking was recorded by illuminating the eye with a Haag-Streit slit-lamp (SL-990). Light was projected on the left eye at $45^{\circ}$ to the nasal part. The images were recorded with a high Speed camera (X_PRI AOS technologies AC) working at $500 \mathrm{fps}$ and a spatial resolution of $800 \times 560$ px situated outside the lamp at $45^{\circ}$ to the left temporal part. $722 \times 541 \mathrm{~mm}(72 \times 72 \mathrm{DPI})$ 


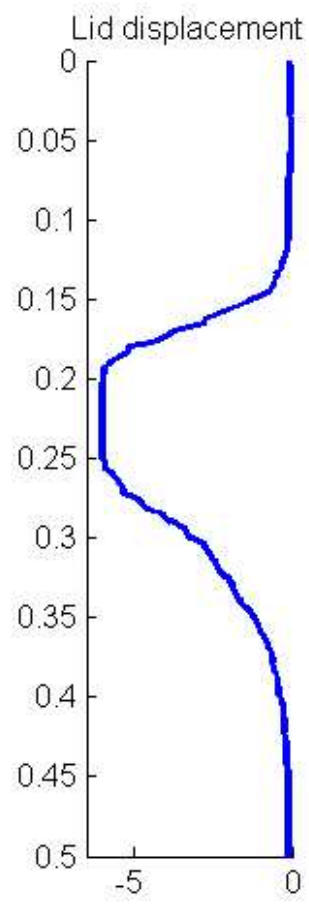

\section{Eve retraction}
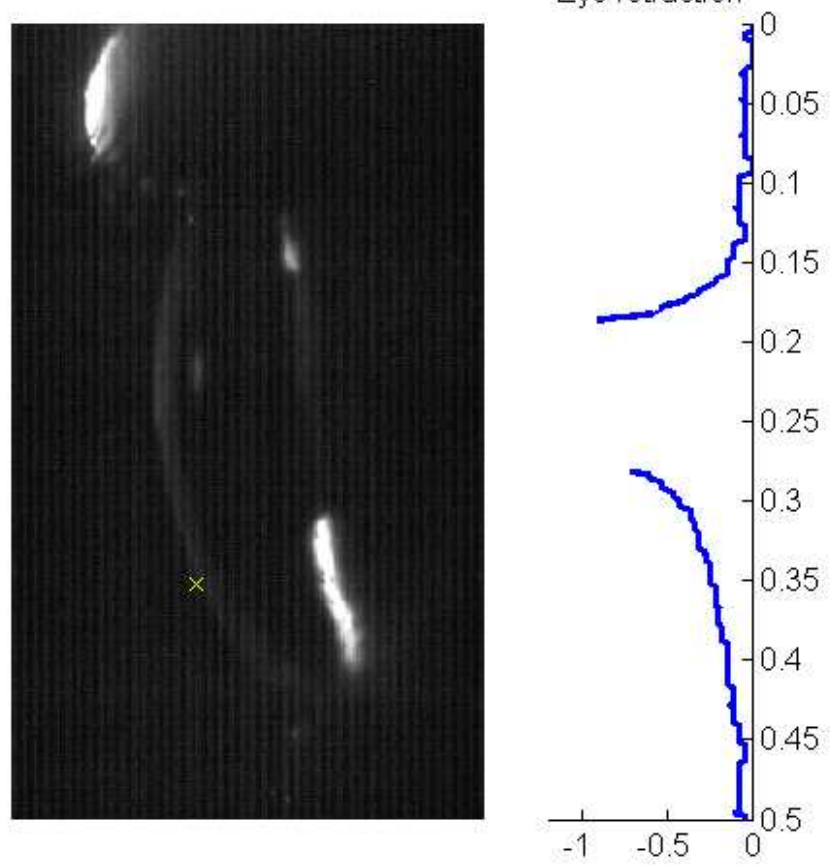

Figure 2 (link to multimedia file). Animation showing the eyelid movement together with the eye retraction. The cross marks the initial position of the cornea. Graphs in the sides show the position (in $\mathrm{mm}$ ) of both elements $28 \times 21 \mathrm{~mm}(600 \times 600 \mathrm{DPI})$ 


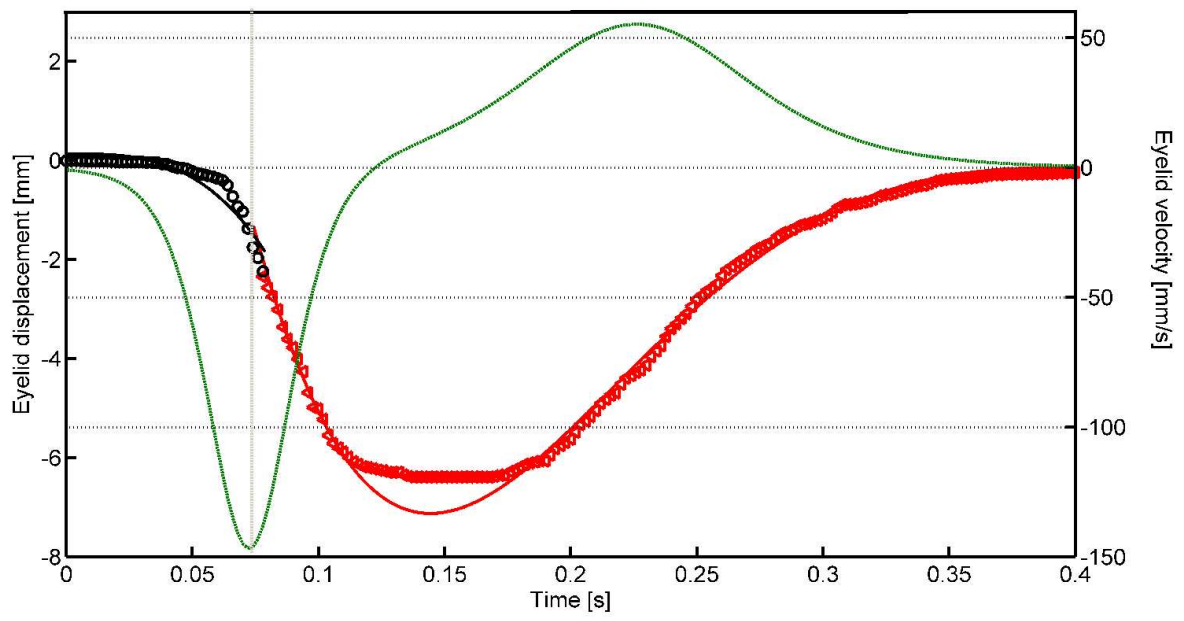

FIGURE 3 Eyelid position and fitting to the model for one typical case. Dashed line shows eyelid velocity during blinking. The vertical line shows the instant where the maximum absolute velocity is achieved being this point the transition between the active phase and the recovery phase.

$280 \times 143 \mathrm{~mm}(600 \times 600 \mathrm{DPI})$ 
FIGURE 4 Anterior corneal position and fitting to the model for one typical case. The vertical dashed line marks the link between the two fitting curves corresponding to the active and the recovery phases.

$280 \times 150 \mathrm{~mm}(600 \times 600 \mathrm{DPI})$ 


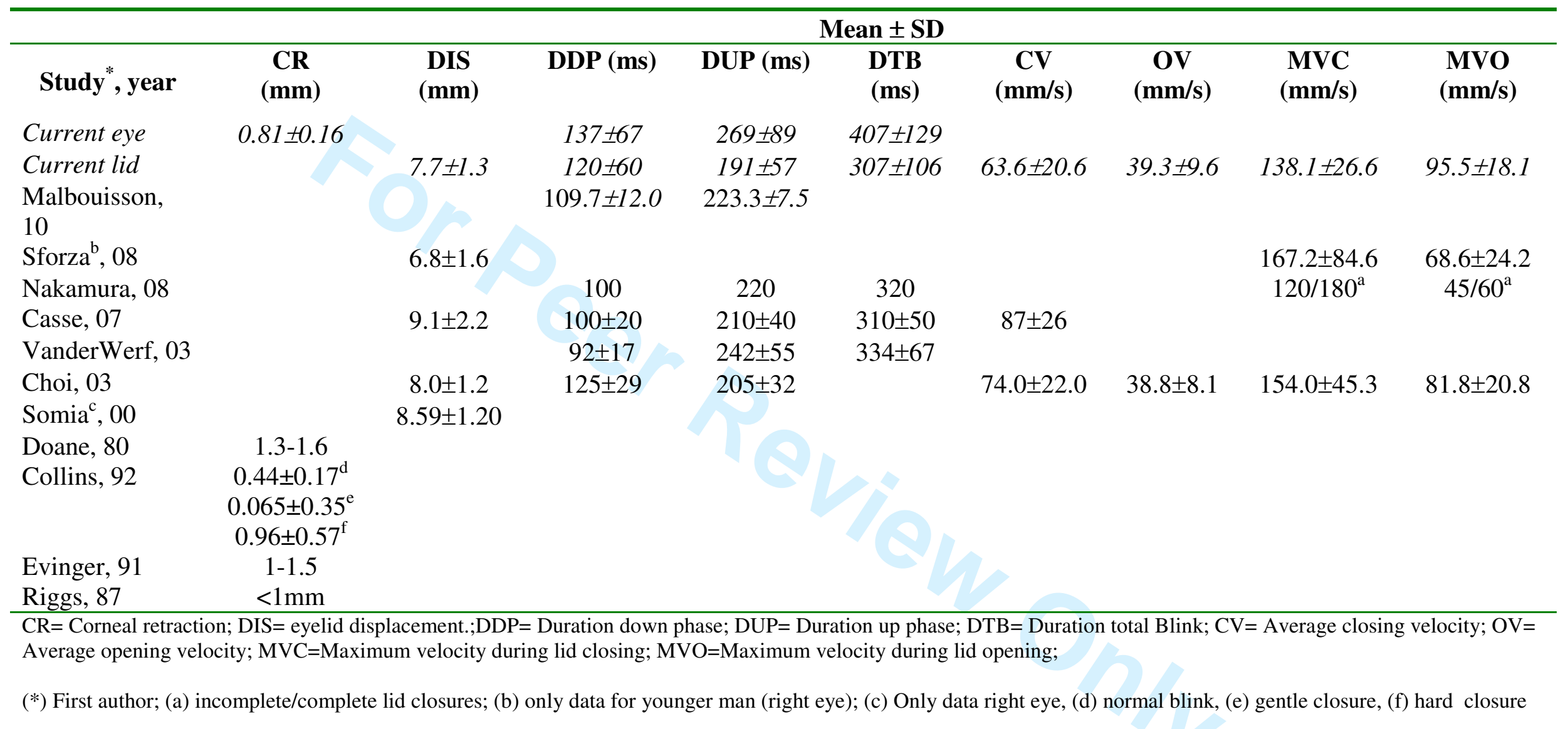

